\title{
The "Sarcopenia and Physical fRailty IN older people: multi- componenT Treatment strategies" (SPRINTT) project: advancing the care of physically frail and sarcopenic older people
}

\author{
Roberto Bernabei $^{1} \cdot$ Luca Mariotti $^{1} \cdot$ Philippe Bordes $^{2} \cdot$ Ronenn Roubenoff $^{3}$
}

Received: 18 September 2016 / Accepted: 4 October 2016 / Published online: 31 January 2017

(C) Springer International Publishing Switzerland 2017

The absolute and relative number of people aged $65+$ is on a sharp rise worldwide [1]. Such a demographic transition is certainly a desirable result of the magnificent combination of socioeconomic development, technological, and medical advancements, and the establishment of solid public health systems. On the other hand, population aging brings forth substantial challenges for healthcare providers, policy makers, and regulators [2]. Indeed, existing healthcare systems, built around the traditional "standalone disease medicine" paradigm, are not equipped to address the complex medical needs of a growing share of the population characterized by multimorbidity and disabilities [3]. As a consequence, a substantial number of older adults do not receive the health services they would need for maximizing their function and quality of life [4]. At the same time, the suboptimal use of resources results in escalating costs which poses a serious threat to the sustainability of health and social care systems. To further complicate the matter, the existence of regulatory gaps hampers the development of innovative strategies in geriatric medicine, as acknowledged by major governmental institutions, including the European Commission [5]. The lack of adequate funding for developing and testing novel interventions as well as the insufficient understanding of their "real life"

Roberto Bernabei

roberto.bernabei@rm.unicatt.it

1 Department of Geriatrics, Neurosciences and Orthopedics, Center for Geriatric Medicine (CeMI), Institute of Internal Medicine and Geriatrics, Catholic University of the Sacred Heart School of Medicine, L.go F. Vito 1, 00168 Rome, Italy

2 Strategy Science Policy and External Innovation, SanofiAventis R\&D, Chilly-Mazarin, France

3 Musculoskeletal Translational Medicine, Novartis Institutes for Biomedical Research, Basel, Switzerland translation represents other major barriers to innovations in geriatrics [5].

The Innovative Medicines Initiative-Joint Undertaking (IMI-JU), a public-private partnership between the European Union and the European Federation of Pharmaceutical Industries and Associations (EFPIA), is strategically equipped to foster the research in areas where there is an unmet medical or social need by supporting collaborative efforts of academic and industrial partners (https:// www.imi.europa.eu/). Within the ninth call for proposals launched by IMI-JU in 2013, the agency identified physical frailty and sarcopenia (PF\&S) as prototype geriatric conditions on which to leverage for advancing the care of older people with unmet needs. The "Sarcopenia and Physical fRailty IN older people: multi-componenT Treatment strategies" (SPRINTT) project has been designed to produce significant advancements in the management of older persons with PF\&S by promoting a consensus among academia, regulators, industry, and patients' representatives over (1) a clear operationalization of frailty; (2) the identification of a precise target population with unmet medical needs; (3) the evaluation and validation of a new methodology for implementing in Europe preventive and therapeutic strategies among frail elders at risk of disability; (4) the definition of an experimental setting serving as template for regulatory purposes and pharmaceutical investigations; and (5) the identification of biomarkers and health technology solutions to be implemented in clinical practice [6]. To ensure the successful accomplishment of the project goals, a consortium has been established convening experts in PF\&S, trial methodology, biomarker discovery and qualification, health technology assessment, information and communication technology (ICT) solutions, regulatory affairs, and dissemination. The consortium is organized in multiple interacting work-package teams, reassembling 
academia, EFPIA, and two small-and-medium-sized enterprises. Each partner has been supporting the conduct of SPRINTT through its own specific expertise. The core of the SPRINTT project is a phase III, multicenter, randomized controlled trial (RCT) aimed at comparing the efficacy in preventing mobility disability of a multi-component intervention, based on long-term structured physical activity, nutritional counseling/dietary intervention, and an ICT intervention, versus a healthy aging lifestyle education program in 1500 older persons with PF\&S.

This issue of Aging Clinical and Experimental Research presents the state of the art of research in the fields of frailty and sarcopenia [7-17]. A specific contribution illustrates the rationale that guided the operationalization of PF\&S by the SPRINTT consortium [18]. The last contribution describes the SPRINTT RCT design and methodology [19]. It is noteworthy that the articles included in this issue result from the collaborative effort of academic and industrial partners. Different backgrounds, views, and approaches have converged to define a project with the common objective of better understanding the pathophysiological foundations and clinical implications of PF\&S. Ultimately, the project aims at finding new strategies for preventing disability and improving the health status in frail older persons.

Acknowledgements The present work was funded by a grant from the Innovative Medicines Initiative-Joint Undertaking (IMI-JU 115621).

\section{References}

1. The World Bank Group (2016) http://data.worldbank.org/indicator/SP.POP.TOTL. Accessed 28 Oct 2016

2. Christensen K, Doblhammer G, Rau R, Vaupel JW (2009) Ageing populations: the challenges ahead. Lancet 374:1196-1208. doi:10.1016/S0140-6736(09)61460-4

3. Cesari M, Marzetti E, Thiem U, Pérez-Zepeda MU, Abellan Van Kan G, Landi F, Petrovic M, Cherubini A, Bernabei R (2016) The geriatric management of frailty as paradigm of "The end of the disease era". Eur J Intern Med 31:11-14. doi:10.1016/j. ejim.2016.03.005

4. Marzetti E, Sanna T, Calvani R, Bernabei R, Landi F, Cesari M (2016) Brand new medicine for an older society. J Am Med Dir Assoc 17:558-559. doi:10.1016/j.jamda.2016.02.024

5. EU (2011) Synthesis report on the public consultation on the European Innovation Partnership on Active and Healthy Ageing. https://ec.europa.eu/research/innovation-union/pdf/activehealthy-ageing/consultation/consultation_report.pdf. Accessed 28 Oct 2016

6. Marzetti E, Calvani R, Landi F, Hoogendijk E, Fougère B, Vellas B, Pahor M, Bernabei R, Cesari M (2015) Innovative medicines initiative: the SPRINT project. J Frailty Aging 4:207-208. doi:10.14283/jfa.2015.69
7. Cesari M, Marzetti E, Calvani R, Vellas B, Bernabei R, Bordes P, Roubenoff R, Landi F, Cherubini A, for the SPRINTT Consortium (2017) The need of operational paradigms for frailty in older persons: the SPRINTT Project. Aging Clin Exp Res. doi:10.1007/s40520-016-0712-5

8. Marzetti E, Calvani R, Tosato M, Cesari M, Di Bari M, Cherubini A, Collamati A, D'Angelo E, Pahor M, Bernabei R, Landi F, on behalf of the SPRINTT Consortium (2017) Sarcopenia: an overview. Aging Clin Exp Res. doi:10.1007/s40520-016-0704-5

9. Tosato M, Marzetti E, Cesari M, Savera G, Miller RR, Bernabei R, Landi F, Calvani R (2017) Measurement of muscle mass in sarcopenia: from imaging to biochemical markers. Aging Clin Exp Res. doi:10.1007/s40520-016-0717-0

10. Calvani R, Marini F, Cesari M, Tosato M, Picca A, Anker SD, von Haehling S, Miller RR, Bernabei R, Landi F, Marzetti E, for the SPRINTT Consortium (2017) Biomarkers for physical frailty and sarcopenia. Aging Clin Exp Res. doi:10.1007/ s40520-016-0708-1

11. Marzetti E, Calvani R, Tosato M, Cesari M, Di Bari M, Cherubini A, Broccatelli M, Savera G, D'Elia M, Pahor M, Bernabei R, Landi F, on behalf of the SPRINTT Consortium (2017) Physical activity and exercise as countermeasures to physical frailty and sarcopenia. Aging Clin Exp Res. doi:10.1007/ s40520-016-0705-4

12. Cruz-Jentoft AJ, Kiesswetter E, Drey M, Sieber CC (2017) Nutrition, frailty and sarcopenia. Aging Clin Exp Res. doi:10.1007/s40520-016-0709-0

13. Vollenbroek-Hutten M, Jansen-Kosterink S, Tabak M, Feletti LC, Zia G, N'dja A, Hermens H, for the SPRINTT Consortium (2017) Possibilities of ICT-supported services in the clinical management of older adults. Aging Clin Exp Res. doi:10.1007/ s40520-016-0711-6

14. Blasimme A (2017) Physical frailty, sarcopenia and the enablement of autonomy: philosophical issues in geriatric medicine. Aging Clin Exp Res. doi:10.1007/s40520-016-0714-3

15. Le Lain R, Ignaszewski C, Klingmann I, Cesario A, de Boer WI, for the SPRINTT Consortium (2017) SPRINTT and the involvement of stakeholders: strategy and structure. Aging Clin Exp Res. doi:10.1007/s40520-016-0706-3

16. Del Signore S, Roubenoff R (2017) Physical frailty and sarcopenia $(\mathrm{PF} \& \mathrm{~S})$ : a point of view from the industry. Aging Clin Exp Res. doi:10.1007/s40520-016-0710-7

17. Sirven N, Rapp T, Coretti S, Ruggeri M, Cicchetti A (2017) Preventing mobility disability in Europe: a health economics perspective from the SPRINTT study. Aging Clin Exp Res. doi:10.1007/s40520-016-0713-4

18. Cesari M, Marzetti E, Calvani R, Cherubini A, Di Bari M, Kortebein P, Del Signore S, Le Lain R, Vellas B, Pahor M, Roubenoff R, Bernabei R, Marzetti E, for the SPRINTT Consortium (2017) Rationale for a preliminary operational definition of physical frailty and sarcopenia in the SPRINTT trial. Aging Clin Exp Res. doi: 10.1007/s40520-016-0716-1

19. Landi F, Cesari M, Calvani R, Cherubini A, Di Bari M, Bejuit R, Mshid J, Andrieu S, Sinclair AJ, Sieber CC, Vellas B, Topinkova E, Strandberg T, Rodriguez-Manas L, Lattanzio F, Pahor M, Roubenoff R, Cruz-Jentoft AJ, Bernabei R, Marzetti E, on behalf of the SPRINTT Consortium (2017) The "Sarcopenia and Physical fRailty IN older people: multi-componenT Treatment strategies" (SPRINTT) randomized controlled trial: design and methods. Aging Clin Exp Res. doi:10.1007/s40520-016-0715-2 